\title{
Medicaid reimbursement fees and its effect on the provision of care
}

\begin{abstract}
The purpose of this project is to determine the effect of Medicaid reimbursement fees on physician practices. Related literature was accessed and incorporated in the document to provide information on the elements that affect physicians and Medicaid enrollees. For a better comprehension, a couple of studies with real examples are also provided for the reader. After analyzing the findings, it is clear that the Medicaid program does not satisfy physician's expectations, as the fees are not enough to cover medical costs in the majority of the cases. Furthermore, research was conducted to address the challenges perceived by clinicians and consumers to identify potential solutions and the impact of such challenges on the quality of services offered by providers of Medicaid plans. The recommendations provided at the end of the document suggest a revision of the fees and the adoption of a model that benefits both physicians and patients alike.
\end{abstract}

Keywords: medicaid, reimbursement, access to care, health care providers, insurance
Volume 2 Issue I - 2015

\author{
Richard Blanco Topping \\ Director, Loma Linda University, USA
}

Correspondence: Richard Blanco Topping, Director, Loma Linda University, I 634 Laurelwood Ave, Colton, CA 92329, USA Tel (909) 9653032, Email richardbo7@gmail.com

Received: January 07, 2015 | Published: January 2I, 2015
Abbreviations: PACA, patient affordable care act; CMS, centers for medicaid and medicaid; CHIP, children health insurance program; AIDS, acquired immunodeficiency syndrome; NC, North Carolina

\section{Introduction}

The selection of the research topic was to have an in-depth analysis of the U.S. health care reimbursement system; specifically, on the relationship between Medicaid services and their effect on the reimbursement process for physicians. In addition, it is interesting to acknowledge the role health care insurance plans play on the quality of care provided. As well, throughout the document both participants (physicians and patients) will be described to determine the factors that influence one or the other in order to provide valid recommendations that will be useful to maintain an appropriate service and create the required change. As George ${ }^{1}$ indicated, the design of health system needs to change from a transactional attitude to a quality rewarding system. The latter applies to the entire industry, as patients are the main reason why health care services exist. That is, the Patient Affordable Care Act (PACA) focus should be characterized by influencing the level of care provided to patients. Moreover, while access to care is vital, the improved outcome should be a core focus.

Additionally, an explanation of the health care environment will be discussed to have a better idea of the effects of Medicaid patients on the service provided by physicians and health care organizations. The governing policies and their implication for the parties will outline some of the benefits and challenges that exist from deferent viewpoints. Not to end without a proposal on what actions may be necessary to improve the conditions, a number of recommendations are also provided with the intention to support the initiatives of access to care for low-income individuals, children, seniors, and disabled individuals. In addition, a number of potential activities that will enhance the quality of service for Medicaid patients and increase physicians' participation in the government plan by adding incentives based on performance.

\section{Background}

\section{Medicaid History}

This section will provide historical information on the inception of Medicaid and the main modifications that occurred with the program over the years. As Patrick \& Freed $^{2}$ noted, they are changes in the national demography that influence Medicaid expansion. As the program was first created to provide insurance coverage for children, seniors and individuals with physical constraints in the poverty level, one of the main challenges in the provision of health insurance for qualified individuals is the reimbursement fee that physicians receive once the service is provided. Due to the low cost or no cost for lowincome individuals, many have not taken advantage of the opportunity due to negative perceptions with public services. ${ }^{3}$

The Centers for Medicaid and Medicaid (CMS) programs were established in the mid 1960s with the objective of providing health care insurance plans for low-income individuals. Two programs were created; Medicare and Medicaid, to serve populations that seek access to care, but did not have the means to satisfy such desire. ${ }^{2}$ Since its inception, Medicaid has gone through many fundamental changes in order to continue to aid individuals with few resources. In addition, the expansion of Medicaid from children, seniors and individuals with limited physical mobility to incorporate a separate child coverage program named Children Health Insurance Program (CHIP) and patients with breast cancer and Acquired Immunodeficiency Syndrome (AIDS) is an example of the necessity of providing access to care to diverse populations.

As might be imagined, many factors impact the healthcare industry, including funding, enrolment and provision of care for Medicaid patients. In addition, despite the efforts to ensure lowincome enrollees access to care there is a period in which a reduction in registration was noticed and the source for such behavior could be low reimbursement fees, restrictions to Medicaid plans and the creation of new rules to ensure efficiency. ${ }^{4} \mathrm{Next}$, as the program faces many challenges there is a continuous effort by Federal, state and local governments to guarantee access to care under the Medicaid 
plan to millions of people who are seeking the services, but fell short for reason that are discussed further within the document.

\section{Challenges}

Since Medicaid is a government and state funded program the challenge is to continue to provide services or plans for individuals that comply with the requirement in times of economic distress. ${ }^{2}$ Therefore, in tough economies programs suffer from a lack of funding, services are reduced based on a limited amount of resources, mainly financial and personnel availability. According to Adams \& Herring ${ }^{4}$ the main concern for Medicaid program is to increase access to physicians' offices; due to little compensation or low reimbursement fees, many physicians are reluctant to accept patients that are part of the program. Moreover, another factor that complicates the Federal and state efforts to increase enrolment and attention is the accessibility to physicians or hospitals facilities from poor communities to locations with higher socioeconomic status. The latter is a challenge that poorest neighborhoods encounter, due to their low-income status. Consequently, as mentioned by Adams \& Herring, ${ }^{4}$ the inclusion of Medicaid enrollees into health maintenance organization between the years of 1990 and 2000 was a strategy to augment more enrollees to doctors' offices.

Another is the challenge to fund different programs when there are changes in the demography. ${ }^{2}$ The main reason the Medicaid program was created in 1965 was to protect children by providing a safe and healthy environment. Overtime, such conditions changed. During the inception of the program, the percentage of children in the nation was almost $40 \%$, while during the mid 2000 s that percentage decreased to just over $24 \%$. Therefore, such numbers justified a shift in funding under the Medicaid program and the creation of additional legislation to support the delivery of care for seniors

\section{Medicaid policies}

\section{Patients}

Access: There are specific guidelines established by CMS regarding access to health care services for qualified individuals, in many cases states have developed their own specifications. Likewise, insurance plans and their benefits are unique from one state to the other. The inception of PACA provided an open market that enables individuals to acquire care based on their particular qualifications and interests. Certainly, the challenges for Medicaid patients are the diversified policies that exist across states. ${ }^{5}$ Currently, it is the same environment for many low-income or disabled individuals. Consequently, differences in states policies create a setting that becomes confusing and defiant for the accomplishment of Medicaid objective. While the Federal government and states attempt to provide conditions to benefit Medicaid patients; on the other hand, such endeavors are not appreciated in many cases by medical providers. Therefore, as the plans do not create an interest to physicians and other providers of care, services to Medicaid populations are shifted to attend other attractive plans.

As Aday ${ }^{6}$ noted, on a national scale health care providers are not willing to work with Medicaid patients. Moreover, Cunningham $\&$ Nichols ${ }^{6}$ pointed out a number of circumstances that restraint's physicians from providing care: i. The line of service provided

ii. Provider's ethnic/racial group

iii. Patient financial status

The previous areas characterize the behavior of physicians' selectivity about which patients will be seen or not. Therefore, the authors continued to mention that the attendance of Medicaid patients shifted from individual health care providers to grouped services provided by larger agencies.

Insurance plans: There are different plans in the market from which individuals are able to access. For instance, Adams \& Herring ${ }^{4}$ studied the effects of profit making options against Medicaid plan to determine the degree of acceptance of Medicaid among physicians. The researchers' study concluded that they are variations, which are associated with the plan offered. In other words, the plans' characteristics vary and may be attractive in some areas and not so attractive in others for physicians' offices. Moreover, Hurley \& Kaiser Commission ${ }^{4}$ continue to mention that despite participation in the plans there was no indication that the visits to clinical services augmented; similarly, the same was noticed for prevention or inpatient services. The latter is another case that is related to the right to provide care to individuals with low-income status.

Benefits: Although Medicaid was created to satisfy the need for coverage among the poor and at the same time provide physicians with incentives to attend the population, the reality is that either side, both physicians and Medicaid enrollees have not perceived great benefits in the provision of health care services and financial retributions. ${ }^{4,7}$ For that reason, patients do not perceive Medicaid services as of great quality as it is seen as a charitable service. More, providers of care do not invest many resources into Medicaid patients' services, as the retribution for such care is not enough to cover cost.

Furthermore, on its website, Medicaid.gov ${ }^{8}$ classifies the program benefits into obligatory and elective benefits. The offering by states differs as each decides which one of the elective benefits is available for their citizens. The list of obligatory services includes general offerings from hospitals, home health, physicians and Federal among others. The elective services are specific benefits that are available based on states eligibility. The list includes, but not limited to dental, spectacles, hospice, chiropractic, and respiratory.

\section{Medicaid impact on physicians' practices}

\section{Reimbursement}

\section{Cost}

While access to Medicaid services increases due to PACA's mandate the underlying issue physicians do not want to work with Medicaid patients for various reasons was not taken care of and unlikely, many more Medicaid enrollees will experience the same lack of faith. According to Feldstein, ${ }^{9}$ there are other organizational issues that Medicaid program needs to review in order to increase its efficiency. For instance, the association between the program's participants and yearly expenses does not match.

Furthermore, in the year 2008, almost $50 \%$ of children were registered for Medicaid services and at the end of the year physicians 
saw only $20 \%$. Similar disparities were seen with other categories within the demographic groups; such differences may need to be revisited to allocate funds with the objective of aligning the resources with the current demand for care. Such process may assist with the formulation of the program budgets for subsequent periods. Likewise, Temkin Greener et al., ${ }^{10}$ commented, that, in the case of nursing homes hospitalizations in the state of New York during the early 2000s, the cost of Medicaid was little less than \$200million for events that were preventable. Still, another report two years later discovered that the cost for returning patients for hospitalization was close to $\$ 5$ million. The latter demonstrates that there are quality issues that increase the cost and at the same time deprive enrollees the opportunity to a quality service.

\section{Fees}

The main complaint given by physicians or health care providers is related to Medicaid reimbursement fees provided by the Federal government and the states. Moreover, this condition has created a negative effect for minority groups, as their power to purchase health care is lower than the cost of providing the service. In addition, previous research indicated that, in many cases, Medicaid coverage would be rejected by providers of care due to financial concerns originated from the plans low cost. ${ }^{6}$ For instance, reimbursement fees does not cover the operational cost resulted from providers' intervention; that helps to lower the quality of care provided to the poor.

Decker $^{11}$ concluded that certain states provide higher reimbursement fee for Medicaid patients to physicians. Consequently, as the fees are higher, medical practitioners find it attractive and as a result, the number of practitioners increases with the acceptance of additional patients. The author insisted that much of the problem have to do with the difference in the reimbursement fees paid by Medicaid. A recent study from North Carolina (NC) Medicaid program reported the response of the sensitivity from nearly 170 orthodontists on their participation in the Medicaid program. ${ }^{12}$ The results demonstrated that, for half of the survey participants, Medicaid was a bad business; this was followed by almost $38 \%$ who did not make a profit from their services. Other cases have similar results as NC experience. Surprisingly, about $80 \%$ of doctors refrained from receiving new cases. The question should be what the Federal government is and states are doing to improve the conditions for physicians and patients alike under the Medicaid program?

\section{Care}

Quality: The issue of quality provided by health care providers for Medicaid program has been in most part of this document. Although the previous statement is true, quality for Medicaid program has been researched to determine if performance based auto assignment was a strategy to motivate the improvement of the service offered by health care providers. ${ }^{13}$ More, in their study Guthrie et al., ${ }^{13}$ concluded that there was a slight indication of the positive effects of performance based auto assignment on the quality of care provided. In other words, the strategy to appoint Medicaid enrollees to performing plans did not increase (as to create a great difference) the quality of care provided by insurance plans.

Decker ${ }^{11}$ noted that there are evidences that corroborate the existence of individuals who receive low quality of care, which is found between low-income status citizens in the U.S. especially when compared with individuals with higher earnings. In addition, Decker ${ }^{11}$ asserted that individuals with earnings under the poverty level have greater fatality risk than citizens with greater financial resources. Unfortunately, the level of care provided to Medicaid enrollees as noted by Decker, ${ }^{11}$ is similar to the care provided to individuals with higher financial income. However, other external elements have a negative impact on the poor's life expectancy.

\section{Policy implications}

According to Longest, ${ }^{14}$ policy implications related to health care have an effect on diverse communities or organizations. Policy making in the health care industry involves clinical professionals, staff and their patients. The decision-making process ends at the Federal, state and local level within the public administration specifically the Legislative, Executive and Judicial branches. Based on the findings presented in the document on Physicians practices and the effect of Medicaid low fees reimbursements, a list of recommendations is followed with the intention to solve many of the issues experienced by physicians and patients alike. In the previous case, research looks at implications of Medicaid reimbursement fees on physician's services. As Feldstein ${ }^{9}$ noted, the rise in Medicaid fees and the containment of expenditures is an ethical dilemma that seems to have no solutions. For instance, physician's participation in the government's plan resulted in a bad business for the majority of providers. ${ }^{15}$ On the other hand, Medicaid's patients experience with the service provided is not close to the service provided through other plans. Therefore, there is a conflict with the quality of services provided and the dissatisfaction of reduced fees declared by medical practitioners.

Therefore, a list of recommendations is provided with the intention of solving the previous issues; which main objective is to improve the quality of the services provided to patients and aid in the improvement of providers and patients' interactions under CMS Medicaid program:

i. Increase Medicaid fees to be on par with Medicare and require providers that serve the plan to increase the quality of care based on patient's outcomes

ii. Increase Medicaid fees as a strategy to increase the number of doctors available to work with the plan

iii. Increase Medicaid fees to motivate physicians' offices to improve the facility environment and decrease health care inequalities among practices

iv. Develop a national research study that integrates the perceptions of Medicaid patients on the quality of care perceived

v. Separately, develop an instrument to make a national assessment on physicians' experiences with Medicaid plan

vi. Provide incentive for Medical practitioners in order to increase their participation in the program

vii. Use the services of managed care plan to maintain Medicaid costs low as it works in other segments

viii. Assigned new graduates to populated areas with Medicaid enrollees and offer incentives or credits to lower their financial aid loans or pay off their loans after a number of continuous years in service

ix. Create a legislation that allows banks and other financial institutions to lower the interest rate for participating professionals 
x. Evaluation of hospitalizations to determine if they are necessary and prevent as much possible readmissions among members of the plan

xi. Implement an indicator that measures provider's efficiency in the use of Federal and state funds

xii. Provide nonprofit Medicaid providers' financial benefits in exchange of improvement in the quality of care.

\section{Conclusion}

Among various factors that restrict patients' access to quality service are the inequalities that minority groups encounter in physicians' offices. ${ }^{16}$ The researchers acknowledge the important role that payper-performance play in the increase of quality among physicians' services. Conversely, Guthrie et al. ${ }^{13}$ concluded that the quality of care does not improve at the expected rate based on their experience in California. The authors studied the effects of new incentives to increase quantity and not quality of care. Moreover, the message perceived of the information provided throughout the document is the burden that Medicaid is causing as the fees are making hospitals vulnerable and, as a result, operational services are been cut to balance the shortage in revenues. In addition, the impact is dependent on the organization's status be it for profit, not for profit, or governmental. Many of the issues have to do with enacted legislation that provides additional resources for many of the cited business model. There is no doubt that the Federal, states and local governments should find a solution to the issue; although, for instances, it seems to be contradictory; on one side the policy is to maintain the cost for Medicaid down and on the other side the reimbursed funds are not enough. Therefore, a list of recommendations is provided centered in solving the actual problem - with the purpose to initiate a collaborative discussion to resolve the problem.

Furthermore, another factor to consider under the Medicaid program is the fees that health care providers collect for services provided. According to Cai et al., ${ }^{10}$ in the case of nursing homes Medicaid patients are more probable to be hospitalized than other plans. In fact, the program due to provider's individual policies and incentives provides such preference. Unfortunately, Medicaid experience is different across sectors; for instance, in substance abuse programs, Terry Mcelrath et al., ${ }^{6}$ reported that the findings in their analysis revealed that it is provable that $50 \%$ of the time patients with substance abuse problems will not be admitted for treatment. Likewise, those that are admitted in the program are contingent for additional funding to cover costs.

There is no doubt that the reimbursement issue needs to be addressed, as changes in the health care industry are ongoing each year. Due to the environment exposed in this document, it is also recommended to have diverse stakeholders at the same table in national settings. The latter will be instrumental to approach the challenges experienced by providers of care and their patients (decrease cost, improve the quality of care, and increase access to health care facilities), which is different from increasing enrollment to Medicaid program.

\section{Acknowledgements}

None.

\section{Conflict of interest}

The author declares no conflict of interest.

\section{References}

1. George JC, Bass TA. Health Care Reform Bill H. R. 3200-America's Affordable Health Choices Act. J Am Coll Cardiol Intv. 2009;2(10):10281030 .

2. Patrick SW, Freed GL. Intergenerational enrollment and expenditure changes in Medicaid: trends from 1991 to 2005. BMC Health Serv Res. 2012;12:327.

3. Hahn Y. The effect of Medicaid physician fees on take-up of public health insurance among children in poverty. J Health Econ. 2012;32(2):452-462.

4. Adams KE, Herring B. Medicaid HMO penetration and its mix: Did increased penetration affect physician participation in urban markets? Health Serv Res. 2008;43(1 Pt 2):363-383.

5. Ellwood MR, Burwell B. Access to Medicaid and Medicare by the lowincome disabled. Health Care Financ Rev. 1990;1990(Suppl):133-148.

6. Mcelrath TYM, Chriqui JF, McBride DC. Factors related to Medicaid payment acceptance at outpatient substance abuse treatment programs. Health Serv Res. 2011;46(2):632-653.

7. Cunningham PJ, Hadley J. Effects of changes in income and practice circumstances on physicians' decisions to treat charity and Medicaid patients. Milbank Q. 2008;86(1):91-123.

8. Medicaid.gov. Medicaid Benefits. USA: Medicaid.gov; 2014.

9. Feldstein PJ. Health policy issues: An economic perspective. 5th ed. USA: Health Administration Press; 2011.

10. Cai S, Mukamel DB, VeazieP, et al. Hospitalizations in nursing homes: does payer source matter? Evidence from New York State. Med Care Res Rev. 2011;68(5):559-578.

11. Decker SL. Medicaid physician fees and the quality of medical care of Medicaid patients in the USA. Review of Economics of the Household. 2007;5(1):95-112.

12. Im JL, Phillips C, Lee J, et al. The North Carolina Medicaid Program: Participation and perceptions among practicing orthodontists. Am J Orthod Dentofacial Orthop. 2007;132(2):144.

13. Guthrie B, Auerback G, Bindman AB. Health plan competition for Medicaid enrollees based on performance does not improve quality of care. Health Aff (Millwood). 2010;29(8):1507-1516.

14. Longest BB. Health policymaking in the United States. 5th ed. USA: Health Administration Press; 2010.

15. Reschovsky JD, OMalley AS. Do primary care physicians treating minority patients report problems delivering high-quality care? Health Aff (Millwood). 2008;27(3):222-231.

16. Blustein J, Weissman JS, Ryan AM, et al. Analysis raises questions on whether pay-for-performance in Medicaid can efficiently reduce racial and ethnic disparities. Health Aff (Millwood). 2011;30(6):1165-1175. 